\title{
FÜHRUNGSKRÄFTE GEWINNEN
}

DDS - Die Deutsche Schule

113. Jahrgang 2021, Heft 2, S. 132-148

https://doi.org/10.31244/dds.2021.02.02

CC BY-NC-ND 4.0 Waxmann 2021

Colin Cramer, Jana Groß Ophoff, Marcus Pietsch \& Pierre Tulowitzki

Schulleitung in Deutschland

\section{Repräsentative Befunde zur Attraktivität, zu Karrieremotiven und zu Arbeitsplatzwechselabsichten}

\section{Zusammenfassung}

Dieser Beitrag stellt repräsentative Befunde zu Karrieren von Schulleitung in Deutschland vor. Ein knappes Drittel der Lehrpersonen hat bereits über eine Schulleitungsposition nachgedacht, diesen Weg aber vorwiegend aus Gründen der Zufriedenheit mit der pädagogischen Arbeit und der befürchteten Beanspruchung nicht verfolgt. Schulleitungen ergreifen das Amt zuvorderst, um mehr Gestaltungsmöglichkeiten, Verantwortung und Autonomie zu haben. Trotz einer hohen Zufriedenheit mit der Arbeit insgesamt zieht derzeit ein Fünftel der Schulleitungen in Deutschland einen Arbeitsplatzwechsel in Erwägung. Die Befunde werden vor dem Hintergrund des virulenten Mangels an Schulleitungen diskutiert.

Schlüsselwörter: Schulleitung, Attraktivität, Karrieremotive, Arbeitsplatzwechselabsicht

\section{School Principals in Germany}

Representative Findings on Attractiveness, Career Motives and Turnover Intentions

\begin{abstract}
This article reports representative findings regarding the careers of school principals in Germany. Almost a third of teachers already thought about applying for a position as a school principal but decided otherwise, not only because they fear stress and strain, but also because they are content with their current pedagogical work. School principals choose their career first and foremost to widen their creative scope and to gain more responsibility and autonomy. Despite a high level of overall job satisfaction, one fifth of school principals in Germany has a turnover intention. The findings are discussed against the backdrop of the virulent shortage of school principals.
\end{abstract}

Keywords: leadership, attractiveness, career motives, turnover intentions 


\section{$1 \quad$ Einleitung}

Die Relevanz von Schulleitung für die Schulentwicklung und indirekt auch für Leistungen von Schüler*innen wird international (Day \& Sammons, 2013; Leithwood, Harris \& Hopkins, 2020; Leithwood \& Louis, 2012) wie national (Bonsen, 2016; Pietsch \& Tulowitzki, 2017) konstatiert. Angesichts der hohen Relevanz von Schulleitung ist erstaunlich, dass in Deutschland zwar vereinzelt Studien zum Schulleitungshandeln vorliegen (im Überblick z. B. Schwanenberg, Klein \& Walpuski, 2018), doch was das Amt der Schulleitung für Lehrpersonen attraktiv macht, warum Schulleitungen diese Position ergriffen haben und welche Gründe gegen einen Verbleib im Amt sprechen, ist bislang im deutschsprachigen Raum wenig erforscht, obgleich es recht häufig zu Schulleitungswechseln kommt (Pietsch, Tulowitzki \& Hartig, 2019). Dabei galten 2018 z. B. in Baden-Württemberg 251 Schulleitungspositionen als unbesetzt (Kramer \& Bauer, 2019); die dpa ging im Dezember 2019 von deutschlandweit mehr als 1.000 unbesetzten Stellen aus (Sadigh \& dpa, 2019). Vor dem Hintergrund dieses akuten Mangels an Schulleitungen wirft dieser Beitrag im Projekt Leadership in German Schools (LineS2020) die Frage auf: Was macht das Amt der Schulleitung (un)attraktiv und welche Karrieremotive und Arbeitsplatzwechselabsichten haben Schulleitungen?

\section{Forschungsstand}

In Deutschland - wie auch in anderen westeuropäischen Ländern oder den USA - liegt ein Mangel an Bewerbungen für das Amt der Schulleitung vor (Tulowitzki, 2019a). Grundsätzlich nehmen sich Schulleitende in Deutschland als erfolgreich wahr, vor allem im Bereich Administration, weniger in Personalführung oder Organisationsentwicklung (Schwanenberg et al., 2018). Trotz der zunehmend wichtigeren Aufgabe der Schulentwicklung wenden Schulleitende mindestens 30 Prozent ihrer Arbeitszeit für Administration und nur 16 Prozent für Aufgaben rund um Curriculum und Unterricht auf (OECD, 2018, S. 38). Entsprechend gilt in Deutschland das Aufgabenprofil als stark durch Verwaltung geprägt (Huber, Gördel, Kilic \& Tulowitzki, 2016, S. 176; Meyer, Richter, Marx \& Hartung-Beck, 2019). Ebenso gelten Arbeitsbelastung und Burnout-Risiko als hoch (Pont, Nusche \& Moorman, 2008, S. 177). Mehrere Studien verdeutlichen, dass Schulleitende in Deutschland besonderen Belastungen aufgrund struktureller Rahmenbedingungen ausgesetzt sind (z. B. Warwas, 2012; Brauckmann \& Herrmann, 2013; Dadaczynski \& Paulus, 2016). Studien aus den USA und Kanada zeigen wiederum negative Wirkungen der Arbeitsbelastung auf Motivation und Effektivität im Beruf (Federici \& Skaalvik, 2012; Wang, Pollock \& Hauseman, 2018; für Deutschland vgl. Huber, Wolfgramm \& Kilic, 2013). 
Die Motive von Lehrpersonen, sich (potenziell) für oder gegen das Schulleitungsamt $z u$ entscheiden, wurden in Deutschland bislang kaum untersucht. Müller, Hancock, Wang und Stricker (2017) widmeten sich der Thematik in einer internationalen, nicht repräsentativen vergleichenden Befragung (Deutschland - USA). Sie unterschieden drei Faktoren: Schulkultur (z.B. Wunsch nach mehr Beteiligung an Schüler*innenaktivitäten), Supervision/Unterstützung von Mitarbeiter*innen (z.B. Wunsch nach mehr Zeit für Entwicklungsaktivitäten) sowie Vergütung und Sozialleistungen (z.B. Wunsch nach höherer Bezahlung). Vom US-amerikanischen Sample unterschied sich die deutsche Stichprobe im Bedürfnis nach höherem Gehalt und besserer Vorqualifikation.

$\mathrm{Zu}$ zentralen Karrieremotiven von Schulleiter ${ }^{\star}$ innen zählen nach Müller et al. (2017) die (vermeintliche) Hoffnung, Schule gestalten, Einfluss auf die Lernchancen von Schüler ${ }^{\star}$ innen nehmen und Lehrpersonen unterstützen zu können - verbunden mit der Hoffnung auf mehr Autonomie und eine Besserstellung der eigenen Position (Hancock, Hary \& Müller, 2012; Hancock, Müller \& Wang, 2016; Hancock, Müller, Wang \& Hachen, 2019). Die US-amerikanischen Befunde lassen sich verkürzt mit dem Slogan „Deciding to lead: Loving the mission and doing it my way“ (Torres, 2018, S. 8) resümieren. Befunde für Deutschland verweisen ebenfalls auf (persönliche) Verbesserung bzw. Entwicklung als zentrales Motiv für das Ergreifen des Amtes (Brauckmann, 2014). So konnte Wissinger (1996) für bayerische Schulleitungen zeigen, dass diese mit ihrer Karriereentscheidung retrospektiv vor allem mehr Verantwortung tragen wollen, die Vielfalt der Leitungsaufgaben attraktiv erscheint und Freude an verwaltungsbezogenen Aufgaben besteht; höheres Einkommen, höhere Reputation und verstärkte Zusammenarbeit mit Erwachsenen werden hingegen als weniger bedeutsam erachtet.

Die Mehrbelastung durch erweiterte Aufgaben in der Schulleitung (z.B. Brauckmann, 2014) wirft zudem die Frage nach Arbeitsplatzwechselabsichten von Schulleiter ${ }^{\star}$ innen bzw. nach Gründen für den Verbleib im Beruf auf. Tran (2017) zeigt anhand US-amerikanischer Daten, dass eine höhere Zufriedenheit mit dem Gehalt die Wahrscheinlichkeit einer Wechselabsicht verringert, während etwa die Schüler ${ }^{\star}$ innenklientel, die Schulgröße oder die Erfahrung der Befragten keinen signifikanten Einfluss haben. Eine Studie von Tekleselassie und Villarreal (2010) unterstreicht die Bezahlung als Einflussfaktor; zusätzlich geraten jedoch Berufszufriedenheit, Autonomieerleben und individuelle Merkmale in den Blick. Boyce und Bowers (2016) unterscheiden zwei Schulleitungstypen, die ihren Arbeitsplatz verlassen: solche, die zufrieden sind und einen Wechsel als Chance sehen, Gehalt, Handlungsspielräume und Herausforderungen zu vergrößern, und die es deshalb aus dem aktuellen Amt zieht („pull“) - und jene Unzufriedenen, die politische Rahmenbedingungen für problematisch erachten, zwischenmenschliche Konflikte und schlechte Arbeitsbeziehungen sehen oder persönliche Gründe anführen, warum sie sich aus dem Amt gedrängt („push“) fühlen. 
Schließlich existieren angesichts der im internationalen Vergleich eher kurzen Qualifizierung von Schulleitungen in Deutschland z.T. beträchtliche Unterschiede zwischen den Bundesländern bezüglich des Verpflichtungsgrades, Umfanges sowie der Modalitäten der Maßnahmen (Tulowitzki, Hinzen \& Roller, 2019). Ebenso stellt sich die berufliche Entwicklung von Schulleitungen, etwa mit Blick auf die einschlägigen Fortbildungsangebote, heterogen dar (Klein \& Tulowitzki, 2020).

\section{Methode}

Die repräsentativen Daten zu Lehrpersonen und Schulleitungen in Deutschland wurden durch den Felddienstleister forsa $\mathrm{GmbH}$ erhoben. Gefördert wurde die Durchführung der Studie durch die Qualitätsoffensive Lehrerbildung von Bund und Ländern aus Mitteln des Bundesministeriums für Bildung und Forschung (FKZ: 01JA1611).

\subsection{Grundgesamtheit, Stichprobe und Durchführung der Studie}

Die Grundgesamtheit der beiden repräsentativen Studien waren Lehrpersonen und Schulleitungen (inkl. Stellvertretungen) an allgemeinbildenden Schulen in ganz Deutschland. Auswahl und Rekrutierung erfolgten telefonisch im Rahmen bevölkerungsrepräsentativer Mehrthemenumfragen. Zur Teilnahme bereite Personen erhielten unmittelbar per E-Mail eine Einladung zur Online-Befragung. Im Rekrutierungsverfahren hatte jedes Mitglied der Grundgesamtheit die gleiche statistische Chance, in die Stichprobe aufgenommen zu werden. Kleinere Abweichungen der Struktur der Stichprobe von der Grundgesamtheit wurden durch Gewichtung (iteratives Verfahren) angeglichen und entsprechende Gewichtungsfaktoren ermittelt. Grundlage hierfür waren Daten des Statistischen Bundesamtes für Lehrkräfte im Schuljahr 2018/2019 an allgemeinbildenden Schulen, die für die Ziel-Verteilung der Stichprobe die Merkmale Alter, Geschlecht, Region (Ost/West) und Schulform vorgaben.

In Summe wurden 1.636 Lehrpersonen in vier in sich repräsentativen Rotationen (Zufallssplits) befragt. Hiervon bilden $n=405$ Lehrpersonen (Befragung: September bis November 2019) die hier analysierte Stichprobe, welche kontrastiert wird mit der Stichprobe von $n=405$ Schulleitungen oder deren Stellvertretungen (Befragung: August bis November 2019). Die Beschreibung der beiden Stichproben in Tabelle 1 auf der folgenden Seite weist die Verteilung nach Alter, Geschlecht, Berufserfahrung, Migrationshintergrund und Schulform aus. 
Colin Cramer, Jana Groß Ophoff, Marcus Pietsch \& Pierre Tulowitzki

Tab. 1: Stichprobenmerkmale der Lehrpersonen und Schulleitungen (in \%)

\begin{tabular}{|c|c|c|c|}
\hline Variable & Kategorie & Lehrpersonen & Schulleitungen \\
\hline \multirow[t]{4}{*}{ Alter } & bis 29 Jahre & 4.7 & 0.0 \\
\hline & 30-44 Jahre & 36.8 & 11.6 \\
\hline & 45-59 Jahre & 42.7 & 56.0 \\
\hline & 60 und mehr Jahre & 15.8 & 32.2 \\
\hline \multirow[t]{2}{*}{ Geschlecht } & weiblich & 65.2 & 55.8 \\
\hline & männlich & 34.8 & 43.7 \\
\hline \multirow{6}{*}{$\begin{array}{l}\text { Berufs- } \\
\text { erfahrung }\end{array}$} & bis 1 Jahr & 1.0 & 0.0 \\
\hline & 1-5 Jahre & 12.3 & 0.7 \\
\hline & 6-10 Jahre & 16.3 & 12.8 \\
\hline & 11-20 Jahre & 33.1 & 28.9 \\
\hline & 20 und mehr Jahre & 35.1 & 64.4 \\
\hline & unsicher bzw. keine Angabe & 2.2 & 1.0 \\
\hline Migration & Migrationshintergrund & 4.7 & 4.2 \\
\hline \multirow[t]{8}{*}{ Schulform } & Grundschule & 23.7 & 51.9 \\
\hline & Hauptschule & 5.7 & 3.0 \\
\hline & Realschule & 11.0 & 9.1 \\
\hline & (Berufliches) Gymnasium & 30.7 & 14.8 \\
\hline & Integrierte Gesamtschule & - & 3.2 \\
\hline & $\begin{array}{r}\text { Schule mit mehreren Bildungsgängen } \\
\text { (z.B. Stadtteilschule oder Gemeinschaftsschule) }\end{array}$ & 19.5 & 8.1 \\
\hline & Sonder- und Förderschule & 8.5 & 6.4 \\
\hline & andere Schulform & 1.0 & 3.5 \\
\hline
\end{tabular}

Anm.: Lehrpersonen: $n=405$; Schulleitungen: $n=405$. Eine (formale) Leitungsposition hatten die Schulleitungen im Schnitt bereits $10.1(S D=7.4)$ Jahre inne; an der spezifischen Schule arbeiteten sie im Mittel bereits $8.7(S D=6.9)$ Jahre.

Quelle: eigene Berechnung

\subsection{Erhebungsinstrumente und Datenanalyse}

Die Online-Fragebögen bestanden aus geschlossenen Fragen, die neben thematisch relevanten Skalen und Items auch demografische Angaben (u.a. Alter, Geschlecht, Schulform, Dienstalter) umfassten und zur Vermeidung von Reihenfolgeeffekten in zufälliger Reihenfolge innerhalb einer Rotation angezeigt wurden. Hierbei wurden überwiegend etablierte Skalen (s. u.) eingesetzt, die durch forsa einem Pretest im Feld unterzogen wurden (je nach Rotation 20-28 Lehrpersonen; 25 Schulleitungen). Die deskriptiven Statistiken wurden mit der Software SPSS 26 berechnet. 
In der Befragung der Lehrpersonen wurde zunächst die Affinität, eine Schulleitungsstelle zu übernehmen, über ein kategoriales Single-Item erhoben: „Haben Sie jemals konkret darüber nachgedacht, sich auf eine Stelle als Schulleiterin oder Schulleiter zu bewerben?" (Antwort: ja, nein, keine Angabe). Weiterhin wurde eine Skala entwickelt zu Gründen, warum sich Lehrpersonen nicht auf eine Schulleitungsstelle bewerben bzw. nicht Mitglied der Schulleitung sind (vgl. Kap. 4.1). Grundlage hierfür war eine Masterarbeit, in welcher Lehrpersonen hierzu interviewt wurden (Nebel, 2016). Aus den Ergebnissen wurden zehn Items abgeleitet, die typische Hindernisgründe (Unattraktivität Schulleitung) operationalisieren. Mittels explorativer Faktorenanalysen (Mplus Version 8; Muthén \& Muthén, 2017; vgl. die Anmerkungen zu Tab. 2 auf S. 139) wurden Modelle unterschiedlicher Dimensionalität über etablierte Goodness-of-Fit-Indizes verglichen (Moosbrugger \& Schermelleh-Engel, 2012). Dabei erwies sich eine dreidimensionale Lösung als die passendste (vgl. Tab. 2), wobei von ursprünglich zehn Items zwei aufgrund keiner eindeutigen Faktorzugehörigkeit bzw. einer Mindestladung $\lambda_{\mathrm{ij}} \leq .30$ auf allen Faktoren ausgeschlossen wurden (Comrey \& Lee, 1992).

Bei der Befragung der Schulleitungen wurden deren Karrieremotive für das Anstreben einer (formalen) Schulleitungsposition mithilfe einer Adaption des revidierten Work Values Inventory (Super, 1970; Zytowski, 2006; Robinson \& Betz, 2008) erhoben. $\mathrm{Zu}$ zehn der dort enthaltenen zwölf Dimensionen wurde je ein Item zur Erhebung der folgenden Bereiche eingesetzt: geistige Herausforderung, Leistung, Kreativität, Altruismus, Abwechslung, Führung Anderer, Sicherheit des Arbeitsplatzes, Unabhängigkeit, Berufsprestige und Einkommen. Ergänzt wurde dies durch eine Frage zu den antizipierten Karrierechancen: „Ich wurde Schulleitung, um meine beruflichen Aufstiegsmöglichkeiten zu verbessern." (vgl. die Anmerkungen zu Abb. 1). Zur Erfassung der Arbeitsplatzwechselabsicht wurde ein Item aus dem US-amerikanischen Schools and Staffing Survey (Boyce \& Bowers, 2016) übernommen: „Wie lange planen Sie, Schulleiterin/Schulleiter an dieser Schule zu bleiben?" (vgl. die Anmerkungen zu Abb. 2). Sofern die Befragten einen Arbeitsplatzwechsel in Erwägung zogen, wurden sie zudem mit Items aus der Studie Berufliche Laufbahnentwicklung von Akademikerinnen und Akademikern der Universität Erlangen-Nürnberg (Abele \& Spurk, 2006) sowie mit zwei ergänzten Items (altersbedingte und gesundheitliche Gründe) nach Gründen für den anvisierten Stellenwechsel befragt (Mehrfachauswahl: nein / ja).

\section{Ergebnisse}

Im Folgenden werden die zentralen Ergebnisse zu Schulleitung in Deutschland mit Blick auf die Aussagen der bevölkerungsrepräsentativen Samples von Lehrpersonen (vgl. Kap. 4.1) sowie Schulleitungen (vgl. Kap. 4.2) dargestellt. 


\subsection{Was Schulleitung für Lehrpersonen unattraktiv macht}

Ein knappes Drittel der Lehrpersonen in Deutschland (30.1\%) hat entsprechend der Angaben in der Befragung bereits konkret darüber nachgedacht, sich auf eine Stelle in der Schulleitung zu bewerben. Empirisch lassen sich drei Dimensionen (vgl. Tab. 2 auf der folgenden Seite) unterscheiden, die das Amt der Schulleitung potenziell unattraktiv machen, nämlich mit dem Amt unvereinbare, andere (pädagogische) Interessen (Dimension 1), Unsicherheit und mangelnde Vorbereitung auf die mit Schulleitung verbundenen Aufgaben (Dimension 2) sowie die erwartete Beanspruchung im Amt und seine geringen Anreize (Dimension 3). Das Modell zeigt gemäß dem Comparative Fit Index (CFI) eine gute, nach dem Root Mean Square Error of Approximation (RMSEA) eine akzeptable Modellpassung. Trotz der vergleichsweise niedrigen Faktorladung (vgl. Kap. 3) des Items „Ich empfinde die formalen Anreize als zu gering [...]" auf dem zugehörigen Faktor wurde es angesichts der inhaltlichen Passung beibehalten. Die Skalen weisen eine für Gruppenvergleiche akzeptable interne Konsistenz auf (Rost, 2013), und es ergeben sich keine signifikanten Schulformunterschiede.

Gegen das Amt der Schulleitung sprechen demnach vor allem die erwartete Beanspruchung und die geringen Anreize, die ihm zugeschrieben werden. Aufgabenfülle und Arbeitsbelastung werden als zu hoch angesehen. Die Rahmenbedingungen der Amtsausübung werden als schwierig erachtet, obwohl formalen Anreizen (z. B. Gehaltssteigerung) vergleichsweise weniger Bedeutung beigemessen wird. Die sich überschneidenden Konfidenzintervalle der Mittelwerte lassen den Schluss zu, dass die Lehrpersonen mit dem Amt der Schulleitung unvereinbare (pädagogische) Interessen als ähnlich bedeutsam erachten. Dagegen scheinen Unsicherheit und mangelnde Vorbereitung auf Aufgaben von Schulleitungen signifikant weniger ins Gewicht zu fallen: Lehrpersonen sind der Auffassung, Aufgaben von Schulleitung recht gut einschätzen zu können. Eher noch sehen sie in der mangelnden Vorbereitung auf die Aufgaben einen Grund, sich nicht für das Amt zu interessieren.

\subsection{Karrieremotive und Arbeitsplatzwechselabsichten von Schulleitungen}

Die zentralen Karrieremotive von Schulleitungen sind in der Retrospektive vor allem der Wunsch, neue Ideen entwickeln und erproben (93\%), eine abwechslungsreiche und kreative Tätigkeit ausüben ( $86 \%$ ), für das Wohl anderer Menschen sorgen (85 \%) sowie eigene Entscheidungen treffen zu wollen (85 \%) (vgl. Abb. 1 auf S. 140). Auch wollen 81 Prozent der befragten Schulleiter*innen anderen Menschen beistehen und helfen. Demgegenüber artikulieren nur 22 Prozent der Schulleitungen, dass ein (sehr) wichtiges Motiv für ihre Berufswahl gewesen sei, ihr berufliches Ansehen verbessern zu wollen, und 27 Prozent gaben an, so ihre weiteren Aufstiegsmöglichkeiten zu erhöhen. Die Steigerung des Einkommens spielte für 36 Prozent der Befragten 
Tab. 2: Gründe von Lehrpersonen gegen die Entscheidung für die Schulleitung

\begin{tabular}{lrrrrr}
\hline Dimension 1: Andere (pädagogische) Interessen & & & & & \\
\cline { 2 - 5 } Item & $M$ & $S D$ & $\lambda_{1}$ & $\lambda_{2}$ & $\lambda_{3}$ \\
\hline $\begin{array}{l}\text { Ich habe berufliche Interessen, die sich nicht mit Schullei- } \\
\text { tung verbinden lassen (z. B. primäres Interesse am Unter- } \\
\text { richten). }\end{array}$ & 4.8 & 2.1 & $\mathbf{0 . 9 2}$ & -0.00 & -0.11 \\
\hline $\begin{array}{l}\text { Ich habe kein Interesse an zentralen Aufgaben der Schul- } \\
\text { leitung (z. B. Verwaltung, Personalführung, Konfliktmanage- } \\
\text { ment). }\end{array}$ & 4.2 & 2.2 & $\mathbf{0 . 5 6}$ & 0.01 & 0.10 \\
\hline $\begin{array}{l}\text { Ich bin mit meiner Tätigkeit als Lehrperson zufrieden (z. B. } \\
\text { Zufriedenheit mit dem Unterrichten und Arbeitsumfeld). }\end{array}$ & 5.6 & 1.5 & $\mathbf{0 . 5 4}$ & $\mathbf{- 0 . 1 5}$ & 0.01 \\
\hline Skala gesamt (a $=.70)$ & 4.8 & 1.6 & (95\%-KI: 4.7-5.0) \\
\hline
\end{tabular}

Dimension 2: Unsicherheit und mangelnde Vorbereitung auf Aufgaben

\begin{tabular}{lllllll}
\hline Ich bin unsicher, welche Aufgaben sich letztlich mit Schullei- & 3.0 & 1.8 & -0.02 & $\mathbf{0 . 8 9}$ & -0.01
\end{tabular} tung verbinden (z. B. unzureichende Kenntnis der Abläufe).

Ich erachte mich nicht ausreichend für die Aufgaben der Schulleitung vorbereitet (z. B. keine hinreichende Qualifikation).

\begin{tabular}{llll}
\hline Skala gesamt $(a=.65)$ & 3.5 & 1.7 & (95\%-KI: 3.4-3.7)
\end{tabular}

Dimension 3: Erwartete Beanspruchung und geringe Anreize

$\begin{array}{lllllll}\text { Ich erachte die Aufgabenfülle und Arbeitsbelastung von } & & 5.6 & 1.8 & -0.03 & -0.02 & \mathbf{1 . 0 3}\end{array}$ Schulleitungen als zu hoch (z. B. hohe zeitliche Belastung, große Verantwortung, Stress).

Ich sehe die Rahmenbedingungen der Schulleitung als $\begin{array}{lllll}5.4 & 1.7 & 0.22 & 0.09 & \mathbf{0 . 4 6}\end{array}$ schwierig an (z. B. enge Vorgaben aus der Schuladministration).

Ich empfinde die formalen Anreize als zu gering (z. B. Gehaltssteigerung ist zu klein).

$\begin{array}{lllll}4.1 & 2.1 & 0.08 & \mathbf{0 . 5 2} & 0.07\end{array}$

Skala gesamt $(a=.65)$

$5.2 \quad 1.5 \quad(95 \%-K I: 5.1-5.4)$

Anm.: Frage: Inwieweit treffen die folgenden Gründe auf die Tatsache zu, dass Sie sich bislang nicht auf eine Stelle als Schulleiterin oder Schulleiter beworben haben bzw. nicht der Schulleitung angehören? Antwortformat: siebenstufige Skala mit Polbeschriftung ( $1=$ trifft überhaupt nicht zu; $7=$ trifft voll und ganz $\mathrm{zu}$ ). Anmerkungen: $N_{\min }=386$; $M=$ Arithmetisches $\quad$ Mittel; $\quad S D=$ Standardabweichung; $\lambda=$ Faktorladung; $95 \%$ $K I=95 \%$-Konfidenzintervall Mittelwert. Faktorenanalyse: Extraktionsmethode: Maximum Likelihood; Rotationsmethode: Geomin; $\mathrm{Chi}^{2}=18.09 ; \mathrm{df}=7 ; \mathrm{Chi}^{2} / \mathrm{df}=2.6 ; \mathrm{RMSEA}^{2}=$ .063 ; CFI $=.982$.

Quelle: eigene Berechnung

eine wichtige oder sehr wichtige Rolle bei der Entscheidung. Während aus Sicht von Schulleitungen insbesondere intrinsische und autonomiebezogene Motive genannt werden, scheinen extrinsische Motive eine vergleichsweise nebengeordnete Rolle zu spielen. 
Abb. 1: Die fünf wichtigsten Motive von Schulleitungen, sich für den Karriereschritt entschieden zu haben

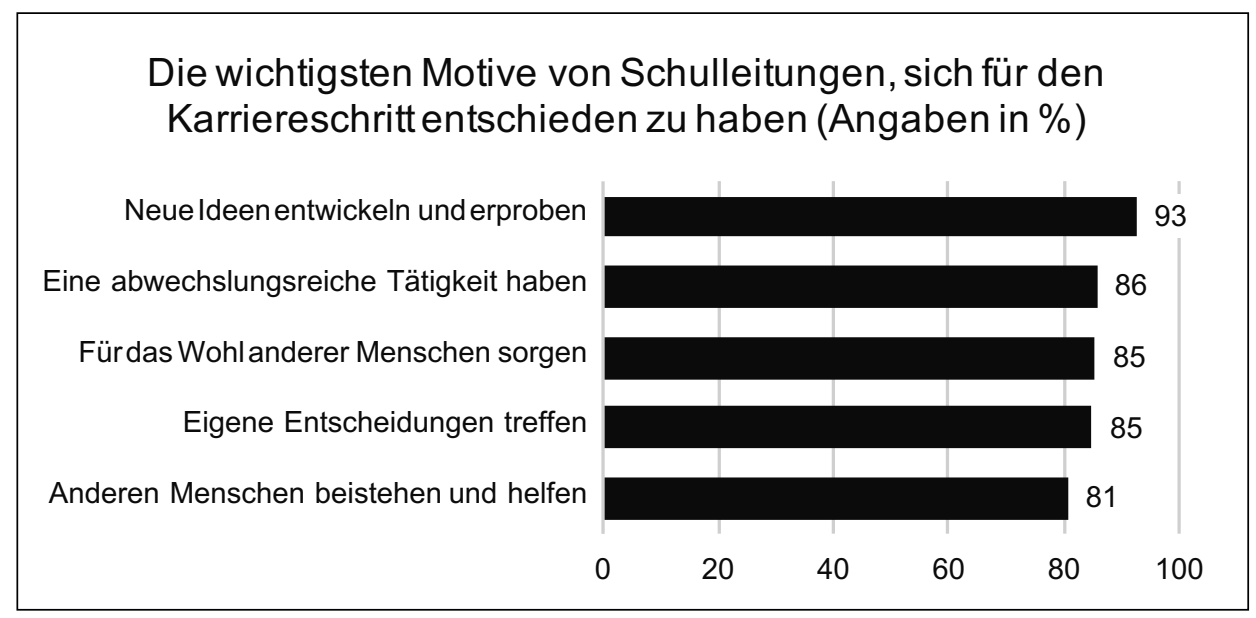

Anm.: Frage: Wie wichtig war der jeweilige Grund für Ihre Entscheidung, Schulleiterin oder Schulleiter zu werden? Antwortformat: Die prozentuale Zustimmung wurde als Summe über die Antwortstufen 3 und 4 gebildet: 1 = sehr unwichtig, $2=$ eher unwichtig, $3=$ eher wichtig, $4=$ sehr wichtig.

Quelle: eigene Darstellung

Mit Blick auf die Arbeitsplatzwechselabsichten von Schulleitungen zeigt sich, dass die befragten Schulleitungen vor Besetzung der aktuellen Stelle im Mittel 2,8mal $(S D=1,91)$ die Stelle gewechselt haben. Dabei beabsichtigt der überwiegende Anteil (67\%), auf der jetzigen Stelle so lange wie möglich zu bleiben. Dennoch planen 15 Prozent der Schulleitungen, die aktuelle Schule zu verlassen, sobald sich eine bessere Möglichkeit ergibt. Vier Prozent geben an, ihren aktuellen Arbeitsplatz so schnell wie möglich verlassen zu wollen. 13 Prozent der Befragten haben hierzu keine Auskunft gegeben. Somit beabsichtigt etwa jede fünfte Schulleitung in Deutschland, (in Zukunft) ihren Arbeitsplatz zu wechseln. Ob für diese Personen eine Schulleitungsposition an einer anderen Schule in den Blick gerät oder ob sie nicht mehr als Schulleiter*in arbeiten wollen, kann auf Basis der Daten nicht differenziert werden. Der Wechselwille variiert zwischen Schulformen (vgl. Tab. 3 auf der folgenden Seite): Schulleitungen an integrierten Gesamtschulen haben eine 71 Prozent geringere Chance, ihren Arbeitsplatz zu wechseln $(O R=0.29)$ als Schulleitungen aller anderen Schulformen. Die Schulleitungen an Hauptschulen hingegen haben eine deutlich stärker ausgeprägte Absicht, ihren Arbeitsplatz zu wechseln, als Schulleitungen an anderen Schulformen $(O R=1.86)$. 
Tab. 3: Arbeitsplatzwechselabsicht nach Schulform

\begin{tabular}{lcc}
\hline Schulform & Wechselabsicht (in \%) & Odds Ratio (OR) \\
\hline Grundschule & 22.4 & 1.17 \\
Hauptschule & 36.4 & 1.86 \\
Realschule & 26.7 & 1.13 \\
(Berufliches) Gymnasium & 16.7 & 0.74 \\
Integrierte Gesamtschule & 7.7 & 0.29 \\
Schule mit mehreren Bildungsgängen & 21.2 & 0.94 \\
(z. B. Stadtteilschule oder Gemeinschaftsschule) & & \\
\hline
\end{tabular}

Anm.: Odds Ratio (OR) geben an, wie hoch die Chance von Schulleitungen einer bestimmten Schulform ist, im Vergleich zu Schulleitungen aller anderen Schulformen ihren aktuellen Arbeitsplatz verlassen zu wollen. Ein Wert von $O R=1$ zeigt keinen Unterschied zwischen den Gruppen, bei $O R>1$ ist die Chance der Arbeitsplatzwechselabsicht größer, bei $O R<1$ geringer.

Quelle: eigene Berechnung

Wird nach Gründen für die Arbeitsplatzwechselabsicht gefragt (vgl. Abb. 2 auf der folgenden Seite), so verweisen wechselwillige Schulleiter ${ }^{\star}$ innen insbesondere auf schwierige Kontextbedingungen. Der zentrale Grund für einen beabsichtigten Arbeitsplatzwechsel ist der Wunsch nach beruflicher Entwicklung (52\%), was etwa jeder zehnten aller Schulleitungen in Deutschland entspricht. 44 Prozent der wechselwilligen Schulleitungen nennen eine nicht angemessene Bezahlung als Motiv, 31 Prozent beklagen zu wenig Unterstützung. 25 Prozent der Schulleitungen nennen Überforderung durch die Arbeitsbedingungen als Motiv der Wechselabsicht, und 24 Prozent weisen auf allgemein schlechte Arbeitsbedingungen hin, während nur zwölf Prozent der Befragten ein generell unbefriedigendes Aufgabenfeld nennen. Der Wunsch nach mehr Zeit für die Familie (19\%) sowie gesundheitliche (15\%) und altersbedingte Gründe (15\%) spielen hingegen eine geringere Rolle. Vergleichsweise selten werden auch Gründe wie ungünstige Arbeitszeiten (7 \%), das Arbeitsklima vor Ort (5\%) oder ein ungünstiger Standort der Schule (5\%) genannt. 
Abb. 2: Die fünf wichtigsten Motive von Schulleitungen, den aktuellen Arbeitsplatz wechseln $\mathrm{zu}$ wollen

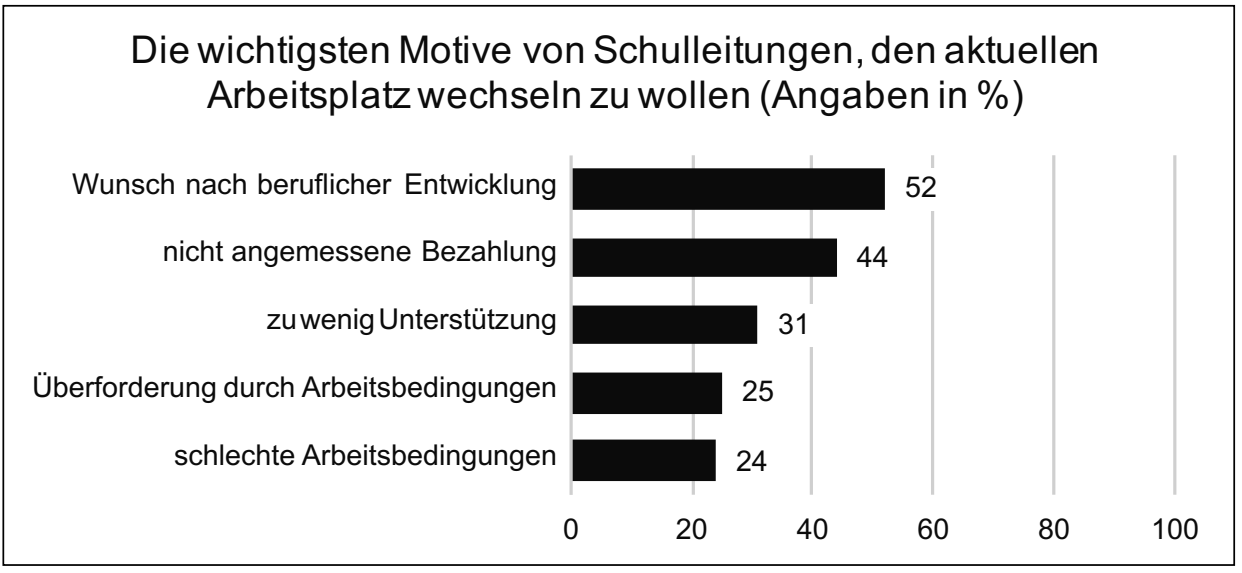

Anm.: Frage: Wie lange planen Sie, Schulleiterin/Schulleiter an dieser Schule zu bleiben? Antwortformat: Die prozentuale Zustimmung wurde als Summe über die Antwortstufen 2 und 3 gebildet: 1 = so lange, wie möglich, 2 = so lange, bis sich mir eine bessere Möglichkeit bietet, 3 = Ich plane definitiv, die Schule so schnell wie möglich zu verlassen, $4=$ kann ich nicht sagen.

Quelle: eigene Darstellung

\section{Diskussion}

Die Befragung von Lehrpersonen konnte zeigen, dass nur ein knappes Drittel bereits einmal konkret darüber nachgedacht hat, Schulleiter*in zu werden. Die wichtigsten Gründe gegen eine Schulleitungskarriere sind insbesondere die mit dem Amt verbundene erwartete hohe Arbeitsbelastung und Aufgabenfülle sowie die Zufriedenheit mit der (Unterrichts-)Tätigkeit als Lehrperson. Auch werden die Rahmenbedingungen, unter denen Schulleitungen arbeiten, als problematisch angesehen. Fehlende (finanzielle) Anreize spielen eine, wenn auch nachgeordnete Rolle.

Der Befragung von Schulleitungen zufolge erweisen sich ein Streben nach Gestaltungsmöglichkeiten, nach Verantwortung für andere und nach Autonomie in der Berufsausübung retrospektiv als zentrale Karrieremotive, während extrinsische Anreize (z.B. ein besseres Gehalt) eine geringere Rolle spielen - was sich überwiegend mit dem eingangs dargestellten Stand der (US-)Forschung deckt. Im Vergleich zu den befragten Lehrpersonen ist für Schulleitungen (also für Lehrpersonen, die den Karriereschritt vollzogen haben) die tatsächlich hohe Belastung im Amt kein maßgeblicher Hinderungsfaktor für ihre Entscheidung. Ob sich damit die Klientel der Lehrpersonen von derjenigen der Schulleitungen auch mit Blick auf Beanspruchungserleben unterscheidet, bleibt eine offene Forschungsfrage. Wenn ein Fünftel der 
Schulleitungen - insbesondere an Hauptschulen - über einen Arbeitsplatzwechsel nachdenken, so tun sie dies insbesondere aufgrund des Wunsches nach beruflicher Weiterentwicklung sowie aufgrund von zu geringer Bezahlung und Unterstützung oder schlechten Arbeitsbedingungen; private bzw. gesundheitliche Motive oder ein generell als unbefriedigend erachtetes Aufgabenfeld fallen dagegen kaum ins Gewicht.

Angesichts dieser repräsentativen Befunde steht infrage, ob alleine die von der Bildungsadministration bislang unternommenen formalen Maßnahmen zur Behebung des Mangels an Schulleitungen (z.B. moderate Erhöhung der Bezüge, Ausbau des Fortbildungsangebots, Werbemaßnahmen) zielführend sind: Entscheidend erscheint, ob die passenden (im Sinne tätigkeitsbezogener Affinität) Lehrpersonen für die Schulleitung gewonnen werden können und inwiefern Entwicklungsmöglichkeiten gegeben sind, die sie im Amt der Schulleitung halten. Offenbar zu wenig wird bislang in Rede gestellt, dass die Beanspruchung im Amt auf Seiten von Lehrpersonen möglicherweise als $(\mathrm{zu})$ bedrohlich wahrgenommen wird und sie zugleich mit ihrer Tätigkeit in Schule und Unterricht zufrieden sind. Es erscheint daher zuvorderst notwendig zu diskutieren, wie Schulleitungen im Amt unterstützt, gefördert und entlastet werden können (z.B. Raum schaffen für Entwicklungsaktivitäten durch höhere Schulautonomie, Delegation von Verwaltungsaufgaben an neu zu schaffende Stellen, Aufwertung der Qualifizierung für Schulleitungen), um für potenziell interessierte (und geeignete) Lehrpersonen attraktiver zu sein. Auch erscheint ein frühzeitigerer Kontakt mit Themen der Leitung und Schulentwicklung auf Organisationsebene sinnvoll, beispielsweise im Lehramtsstudium im Rahmen eines Wahlmoduls, um ein Verständnis für die Organisationsebene zu schärfen und frühzeitig für die Karriereoption Schulleitung zu sensibilisieren. Schulleitungen selbst sind im Amt ebenfalls überwiegend mit ihrem Aufgabengebiet zufrieden, da die Tätigkeit an sich kaum einen Grund für eine Arbeitsplatzwechselabsicht darzustellen scheint.

Keinen Einfluss hingegen hat die Bildungsadministration auf die Berufswahlmotive von Lehrpersonen, die aus ganz spezifischen und vorwiegend pädagogisch-intrinsischen Motiven heraus ihren Beruf ergreifen (Cramer, 2016) und damit kaum einen ausreichenden Pool zur Akquise von geeigneten Schulleitungen darstellen. Solche intrinsischen Motive (wie z.B. das pädagogische Interesse) sind schon zu Beginn des Lehramtsstudiums ausgeprägt (Cramer, 2012; Franz \& Groß Ophoff, 2019), vergleichsweise stabil (Roness \& Smith, 2010; Richardson \& Watt, 2016) und - zumindest aus Sicht der Mehrheit der befragten Lehrpersonen - inkompatibel mit den Tätigkeiten von Schulleitungen. Während der Fit-Choice-Theorie zufolge die Berufswahl von Lehrpersonen ein komplexes Zusammenspiel der Bewertung beruflicher Anforderungen und Erträge unter Einbezug der eigenen Kompetenzerwartung ist (Watt et al., 2012), spielen für Schulleitungen extrinsisch-verlegenheitsbezogene Motive retrospektiv eine geringere bzw. keine Rolle. Mentoring-Programme müssten daher eher dem Abgleich der Motivlagen dienen als zum Ziel zu haben, Lehrpersonen 
das Amt der Schulleitung „schmackhaft“ zu machen; denn sie meinen, die Aufgaben von Schulleitung zu kennen, und nehmen diese ggf. als abschreckend wahr.

Damit gerät die Frage nach einem eigenen und vom Lehramt zu unterscheidenden Berufsstand Schulleitung samt spezifischer Qualifikationswege in den Blick. Nicht beantwortet werden kann allerdings, ob solche Lehrpersonen ihre Affinität im Rahmen ihrer besonderen Aufgaben allererst entwickeln oder ob diese Affinität bereits der Anlass für die Übernahme besonderer Aufgaben ist. Nur im letzteren Fall könnte Schulleitung als ein vom Lehrer*innenberuf entkoppeltes Berufsbild verstanden werden, für das interessierte und geeignete Personen auch außerhalb des Lehrkörpers gewonnen und qualifiziert werden sollten. Plausibel erscheint es vielmehr, vielfältige Wege in den Beruf zu schaffen. Eine eigenständige Qualifizierung vor dem Hintergrund des tatsächlichen Aufgabenprofils könnte Diskrepanzen zwischen Erwartungen an die Schulleitung und dem Berufsalltag verringern. Bislang ist die Schulleitungsqualifizierung in Deutschland nach wie vor zu heterogen und unstrukturiert (Tulowitzki et al., 2019), während Schulleitungen fast vollständig aus dem Lehrpersonal akquiriert werden. Die Rolle von Schulaufsicht und Schulinspektion, die Schulleitungen unterstützen und motivieren können, ist ungeklärt (Heinrich, 2017; Tulowitzki, 2019b).

Die repräsentativen Ergebnisse der Studie zum Thema Schulleitung aus der teils komplementären Sicht von Lehrpersonen und Schulleitungen weisen besonderes Potenzial für die Qualifikationswege von Schulleitung auf. Limitationen ergeben sich u.a. daraus, dass die Karrieremotive von Schulleitungen retrospektiv erhoben wurden, womit die realen Motivlagen zum Zeitpunkt der Karriereentscheidung verzerrt erfasst sein könnten (recall bias; z. B. Schwarz, 2007). Diese Unschärfe kann durch die Befragung von Lehrpersonen zu Hinderungsgründen, eine Schulleitungsposition zu ergreifen, partiell kompensiert werden. Das eigens hierfür entwickelte Instrument zeigt zwar eine akzeptable bis gute Modellpassung, weist aber nur befriedigende interne Konsistenzen in den Subskalen auf. Es sollte daher in künftiger Forschung optimiert (z.B. Entwicklung weiterer Items) und um Aspekte wie Schulkultur und Schulentwicklung (vgl. Müller et al., 2017) erweitert werden, um die Motive von Lehrpersonen und Schulleitungen stärker aufeinander beziehen zu können. Ferner sollte untersucht werden, warum gerade Schulleitungen an Hauptschulen besonders häufig über einen Arbeitsplatzwechsel nachdenken. Solche Analysen werden möglich, weil das Projekt LineS als Längsschnitt angelegt ist. Forschungsdesiderate zeigen sich zudem hinsichtlich der Analyse homogener Subgruppen sowohl seitens Lehrpersonen als auch Schulleitungen. 


\section{Literatur und Internetquellen}

Abele, A. E., \& Spurk, D. (2006). Berufliche Laufbahnentwicklung von Akademikerinnen und Akademikern der Universität Erlangen-Nürnberg (BELA-E). Zugriff am 24.01.2020. Verfügbar unter: https://www.sozialpsychologie.phil.fau.de/files/2016/10/BELA2.pdf.

Bonsen, M. (2016). Wirksame Schulleitung. In H. Buchen \& H.-G. Rolff (Hrsg.), Professionswissen Schulleitung (4., überarb. u. erw. Aufl.) (S. 193-228). Weinheim: Beltz.

Boyce, J., \& Bowers, A. J. (2016). Principal Turnover: Are there Different Types of Principals Who Move from or Leave Their Schools? Leadership and Policy in Schools, 15 (3), 237272. http://dx.doi.org/10.1080/15700763.2015.1047033

Brauckmann, S. (2014). Ergebnisbericht im Rahmen des BMBF Forschungsschwerpunkts "Steuerung im Bildungssystem" (SteBis) geförderten Forschungsprojekts „Schulleitungshandeln zwischen erweiterten Rechten und Pflichten (SHaRP)“. Unter Mitarbeit von C. Herrmann, K. D. Hanßen, S. Böse, T. Holz \& T. Feldhoff. Berlin: DIPF.

Brauckmann, S., \& Herrmann, C. (2013). Belastungserleben von Schulleiterinnen und Schulleitern im Rahmen erweiterter schulischer Eigenständigkeit - Erste empirische Befunde aus der SHaRP-Studie. In I. van Ackeren, M. Heinrich \& F. Thiel (Hrsg.), Evidenzbasierte Steuerung im Bildungssystem? Befunde aus dem BMBF-SteBis-Verbund (DDS - Die Deutsche Schule, 12. Beiheft) (S. 172-196). Münster: Waxmann.

Comrey, A. L., \& Lee, H. B. (1992). A First Course in Factor Analysis. Hillsdale, MI: Lawrence Erlbaum.

Cramer, C. (2012). Entwicklung von Professionalität in der Lehrerbildung. Empirische Befunde $z u$ Eingangsbedingungen, Prozessmerkmalen und Ausbildungserfahrungen Lehramtsstudierender. Bad Heilbrunn: Klinkhardt.

Cramer, C. (2016). Berufswahl Lehramt: Wer entscheidet sich warum? In M. Rothland (Hrsg.), Beruf Lehrer/Lehrerin. Ein Studienbuch (S. 261-276). Münster: Waxmann.

Dadaczynski, K., \& Paulus, P. (2016). Wohlbefinden von Schulleitungen in Deutschland: Ausprägungen und Zusammenhänge mit Arbeit und Gesundheit. Prävention und Gesundheitsförderung, 11 (3), 171-176. https://doi.org/10.1007/s11553-016-0544-3

Day, C., \& Sammons, P. (2013). Successful Leadership: A Review of the International Literature. Zugriff am 23.07.2020. Verfügbar unter: https://files.eric.ed.gov/fulltext/ ED546806.pdf.

Federici, R. A., \& Skaalvik, E. M. (2012). Principal Self-Efficacy: Relations with Burnout, Job Satisfaction and Motivation to Quit. Social Psychology of Education, 15 (3), 295-320. https://doi.org/10.1007/s11218-012-9183-5

Franz, E.-K., \& Groß Ophoff, J. (2019). Kompetenzerleben Lehramtsstudierender im Semesterpraktikum. In T. Ehmke, P. Kuhl \& M. Pietsch (Hrsg.), Lehrer. Bildung. Gestalten. Beiträge zur empirischen Forschung in der Lehrerbildung (S. 116-127). Weinheim: Beltz.

Hancock, D. R., Hary, C., \& Müller, U. (2012). An Investigation of Factors Impacting the Motivation of German and US Teachers to Become School Principals. Research in Comparative and International Education, 7 (3), 352-363. https://doi.org/10.2304/ rcie.2012.7.3.352

Hancock, D. R., Müller, U., \& Wang, C. (2016). Motivating Teachers to Become School Principals in the U.S.A. and Germany. Journal of Global Research in Education and Social Science, 7 (2), 83-93.

Hancock, D. R., Müller, U., Wang, C., \& Hachen, J. (2019). Factors Influencing School Principals' Motivation to Become Principals in the U.S.A. and Germany. International Journal of Educational Research, 95, 90-96. https://doi.org/10.1016/j.ijer.2019.04.004

Heinrich, M. (2017). Zum Verhältnis von Schulinspektion und Schulaufsicht: Zwei Institutionen zwischen Ausdifferenzierung und Entdifferenzierung. In A. Paseka, M. 
Heinrich, A. Kanape \& R. Langer (Hrsg.), Schulentwicklung zwischen Steuerung und Autonomie (S. 155-174). Münster: Waxmann.

Huber, S. G., Gördel, B.-M., Kilic, S., \& Tulowitzki, P. (2016). Accountability in the German School System. In J. Easley II \& P. Tulowitzki (Hrsg.), Educational Accountability International Perspectives on Challenges and Possibilities for School Leadership (S. 165183). London: Routledge.

Huber, S. G., Wolfgramm, C., \& Kilic, S. (2013). Vorlieben und Belastungen im Schulleitungshandeln. In S. G. Huber (Hrsg.), Jahrbuch Schulleitung 2013 (S. 259-271). Köln: Carl Link.

Jong, D. D., Grundmeyer, T., \& Yankey, J. (2017). Identifying and Addressing Themes of Job Dissatisfaction for Secondary Principals. School Leadership \& Management, 37 (4), 354-371. https://doi.org/10.1080/13632434.2017.1338253

Klein, E. D., \& Tulowitzki, P. (2020). Die Fortbildung von Schulleiter*innen in Forschung und Praxis - ein Systematisierungsversuch. DDS - Die Deutsche Schule, 112 (3), 257276. https://doi.org/10.31244/dds.2020.03.02

Kramer, C., \& Bauer, L. (2019). Das Standortnetz von Grundschulen in Baden-Württemberg im Wandel - Entwicklungen und Einflussfaktoren. In D. Fickermann \& H. Weishaupt (Hrsg.), Bildungsforschung mit Daten der amtlichen Statistik (DDS - Die Deutsche Schule, 14. Beiheft) (S. 87-104). Münster: Waxmann. https://doi.org/10.31244/dds. bh.2019.14.06

Leithwood, K., Harris, A., \& Hopkins, D. (2020). Seven Strong Claims about Successful School Leadership Revisited. School Leadership \& Management, 40 (1), 5-22. https:// doi.org/10.1080/13632434.2019.1596077

Leithwood, K., \& Louis, K. S. (2012). Linking Leadership to Student Learning. San Francisco, CA: Jossey-Bass.

Meyer, A., Richter, D., Marx, A., \& Hartung-Beck, V. (2019). Welche Aufgaben haben Schulleitungen heute? Eine Analyse von Schulleitungsaufgaben im innerdeutschen Vergleich. Zeitschrift für Bildungsverwaltung, 35 (2), 23-44.

Moosbrugger, H., \& Schermelleh-Engel, K. (2012). Exploratorische (EFA) und Konfirmatorische Faktorenanalyse (CFA). In H. Moosbrugger \& A. Kelava (Hrsg.), Testtheorie und Fragebogenkonstruktion (S. 325-343). Berlin: Springer.

Müller, U., Hancock, D. R., Wang, C., \& Stricker, T. (2017). Warum fällt es so schwer, sich für das Schulleitungsamt zu entscheiden? Beruf: Schulleitung, 12 (1), 33-35.

Muthén, L. K., \& Muthén, B. O. (2017). MPlus Version 8. Los Angeles, CA: Muthén \& Muthén.

Nebel, L. (2016). Unbeliebter Chefsessel. Eine explorative Untersuchung von hemmenden Faktoren bei der Übernahme von Schulleitungspositionen. Unveröff. Masterarbeit. Tübingen: Universität Tübingen.

OECD (Organization for Economic Cooperation and Development). (2018). TALIS 2018 Results, Vol. I: Teachers and School Leaders as Lifelong Learners. Paris: OECD. https:// doi.org/10.1787/1d0bc92a-en

Pietsch, M., \& Tulowitzki, P. (2017). Disentangling School Leadership and Its Ties to Instructional Practices - an Empirical Comparison of Various Leadership Styles. School Effectiveness and School Improvement, 28 (4), 629-649. https://doi.org/10.1080 /09243453.2017.1363787

Pietsch, M., Tulowitzki, P., \& Hartig, J. (2019). Examining the Effect of Principal Turnover on Teaching Quality: A Study on Organizational Change with Repeated Classroom Observations. School Effectiveness and School Improvement, Advance Online Publication, 1-23. https://doi.org/10.1080/09243453.2019.1672759

Pont, B., Nusche, D., \& Moorman, H. (2008). Improving School Leadership, Vol. 1: Policy and Practice. Paris: OECD. 
Richardson, P. W., \& Watt, H. M. G. (2016). Factors Influencing Teaching Choice: Why Do Future Teachers Choose the Career? In J. Loughran \& M. L. Hamilton (Hrsg.), International Handbook of Teacher Education (S. 275-304). Singapore: Springer.

Robinson, C. H., \& Betz, N. E. (2008). A Psychometric Evaluation of Super's Work Values Inventory-Revised. Journal of Career Assessment, 16 (4), 456-473.

Roness, D., \& Smith, K. (2010). Stability in Motivation during Teacher Education. Journal of Education for Teaching, 36 (2), 169-185. https://doi/10.1080/02607471003651706

Rost, D. H. (2013). Interpretation und Bewertung pädagogisch-psychologischer Studien. Eine Einführung (3. Aufl.). Bad Heilbrunn: Klinkhardt.

Ryan, R. M., \& Deci, E. L. (2017). Self-Determination Theory. Basic Psychological Needs in Motivation, Development, and Wellness. New York: Guilford.

Sadigh, P., \& dpa (2019, 15. Dezember). Schulleiter: Bundesweit fehlen Rektoren in den Schulen. ZEIT online. Zugriff am 06.02.2020. Verfügbar unter: https://www.zeit.de/ge sellschaft/schule/2019-12/schulleiter-mangel-schule-grundschule.

Schwanenberg, J., Klein, E. D., \& Walpuski, M. (2018). Wie erfolgreich fühlen sich Schulleitungen und welche Unterstützungsbedürfnisse haben sie? (SHIP Working Paper Reihe, No. 03). Essen: Universität Duisburg-Essen. https://doi.org/10.17185/duepublico/ 47202

Schwarz, N. (2007). Retrospective and Concurrent Self Reports. The Rationale for Real-time Data Capture. In A. A. Stone, S. Shiffman, A. A. Atienza \& L. Nebeling (Hrsg.), The Science of Real-time Data Capture (S. 11-26). New York: Oxford University Press.

Steiger, J. H. (1990). Structural Model Evaluation and Modification: An Interval Estimation Approach. Multivariate Behavioral Research, 25 (2), 173-180. http://dx.doi.org/10.1207/ s15327906mbr2502_4

Super, D. E. (1970). Work Values Inventory. Boston, MA: Houghton Mifflin.

Tekleselassie, A. A., \& Villarreal, P. I. (2010). Career Mobility and Departure Intentions among School Principals in the United States: Incentives and Disincentives. Leadership and Policy in Schools, 10 (3), 251-293.

Torres, A. C. (2018). Push, Pull, Tap and Switch: Understanding the Career Decisions of Charter School Leaders. Leadership and Policy in Schools, 19 (2), 171-189. https://doi. org/10.1080/15700763.2018.1513155

Tran, H. (2017). The Impact of Pay Satisfaction and School Achievement on High School Principals' Turnover Intentions. Educational Management Administration \& Leadership, 45 (4), 621-638. https://doi.org/10.1177/1741143216636115

Tulowitzki, P. (2019a). School Leader Recruitment, Retention and Motivation in Europe. Desk-Research-Bericht für das European Educational Policy Network (EEPN). http:// dx.doi.org/10.26041/fhnw-2034

Tulowitzki, P. (2019b). Supporting Instructional Leadership and School Improvement? Reflections on School Supervision from a German Perspective. Journal of Educational Administration, 57 (5), 571-581. https://doi.org/10.1108/JEA-03-2019-0040

Tulowitzki, P., Hinzen, I., \& Roller, M. (2019). Die Qualifizierung von Schulleiterinnen und Schulleitern in Deutschland - ein bundesweiter Überblick. DDS - Die Deutsche Schule, 111 (2), 149-170. https://doi.org/10.31244/dds.2019.02.04

Wang, F., Pollock, K., \& Hauseman, C. (2018). School Principals' Job Satisfaction: The Effects of Work Intensification. Canadian Journal of Educational Administration and Policy, 185, 73-90.

Warwas, J. (2012). Berufliches Selbstverständnis, Beanspruchung und Bewältigung in der Schulleitung. Wiesbaden: VS.

Watt, H. M. G., Richardson, P. W., Klusmann, U., Kunter, M., Beyer, B., Trautwein, U., et al. (2012). Motivations for Choosing Teaching as a Career: An International Comparison 
Colin Cramer, Jana Groß Ophoff, Marcus Pietsch \& Pierre Tulowitzki

Using the FIT-Choice Scale. Teaching and Teacher Education, 28 (6), 791-805. http:// dx.doi.org/10.1016/j.tat

Wissinger, J. (1996). Perspektiven schulischen Führungshandelns. Eine Untersuchung über das Selbstverständnis von SchulleiterInnen. Weinheim: Juventa.

Zytowski, D. (2006). Super Work Values Inventory-Revised: Technical Manual (Version 1.0). Zugriff am 01.07.2020. Verfügbar unter: https://www.kuder.com/research/technicalbriefs/supers-work-values-inventory-r/.

Colin Cramer, Prof. Dr., geb. 1979, Lehrstuhlinhaber für Professionsforschung unter besonderer Berücksichtigung der Fachdidaktiken an der Eberhard Karls Universität Tübingen.

E-Mail: colin.cramer@uni-tuebingen.de

Korrespondenzadresse: Eberhard Karls Universität Tübingen, Wilhelmstraße 31, 72074 Tübingen

Jana Groß Ophoff, Prof. Dr., geb. 1976, Professorin für Bildungswissenschaften an der Pädagogischen Hochschule Vorarlberg.

E-Mail: jana.grossophoff@ph-vorarlberg.ac.at

Korrespondenzadresse: Pädagogische Hochschule Vorarlberg, Liechtensteiner Straße 33-37, 6800 Feldkirch, Österreich

Marcus Pietsch, PD Dr., geb. 1974, Professur für Empirische Grundschulpädagogik an der Leuphana Universität Lüneburg.

E-Mail: marcus.pietsch@leuphana.de

Korrespondenzadresse: Leuphana Universität Lüneburg, Universitätsallee 1, 21335 Lüneburg

Pierre Tulowitzki, Prof. Dr., geb. 1982, Leiter der Professur für Bildungsmanagement und Schulentwicklung an der Pädagogischen Hochschule der Fachhochschule Nordwestschweiz.

E-Mail: pierre.tulowitzki@fhnw.ch

Korrespondenzadresse: Pädagogische Hochschule der Fachhochschule Nordwestschweiz, Bahnhofstrasse 6, 5210 Windisch, Schweiz 